\title{
Research on supply chain disruption risk coordination based on combined flexible contract
}

\author{
Yu Jian
}

Xi'an University of Arts and Science, Xi'an, China

\begin{abstract}
Keywords: Quantity Flexible contract, Supply chain coordination, Disruption risk management, Buy-back and revenue sharing contract.
\end{abstract}

\begin{abstract}
This paper studied coordination problem of a three stage supply chain with one retailer one distributors and one manufacturer based on flexible contract composed of repurchase and revenue sharing on supply, when unexpected events have an impact on market demand. Firstly, we adopt the supply chain optimal order quantity under the stable market of no contract constraint and centralized decision; Decentralized decision-marking by combined flexible contract can achieve centralized decision-marking state; The supply chain optimal order quantity under using the original flexible contract is not pareto optimal, when the market demand has a greater impact; Moreover, propose supply chain emergency coordination model by adjusting the contract parameters.
\end{abstract}

\section{基于组合式柔性契约的供应链应急协调管理研究 \\ 余剑}

西安文理学院经济管理学院, 西安, 陕西, 中国, 710065

1411594487@qq.com

关键词：数量柔性契约；供应链协调；应急管理；回购及收益共享契约

中文摘要. 针对一个由零售商、分销商和供应商构成的三级供应链, 当突发事件对市场需求 产生很大冲击时, 研究基于回购与收益共享的组合式柔性契约对供应链系统协调的作用。提 出稳定市场无契约约束时、集中决策下的供应链最优订货量; 分散决策下通过组合式柔性契 约约束可以达到集中决策时的状态; 市场需求发生较大冲击时, 继续使用原有组合式柔性契 约时的最优订货量不是 Pareto 最优; 提出调整契约参数实现供应链应急协调的模型。

\section{1.引言}

随着全球经济一体化的深入发展，企业供应链系统运行的环境变得日益不确定。尤其当 企业外部的不确定性突发事件严重懪发时, 供应链系统的协调性会遭到破坏。在众多供应链 应急协调契约中, 柔性契约是有效减缓供应链风险并促使其应急恢复的重要工具。本文以受 突发事件冲击的三级供应链为研究对象, 对基于回购及收益共享契约的组合式柔性契约在供 应链协调管理中的不同效果进行分析, 通过设定参数变换分析供应链应急协调管理下的组合 式柔性契约模型对供应链系统协调和系统中各成员效率的影响。

\section{2.模型描述}

考虑由一个由占主导地位的生产商 $(\mathrm{S})$ 、分销商 $(\mathrm{D})$ 和零售商 $(\mathrm{R})$ 构成的三级供应 链系统, 分销商及零售商是追随者, 三者都是完全理性和风险中性的, 都各自遵循自身期望 
利润最大化原则，市场没有信息不对称问题。生产商向分销商及零售商提供单一产品,产品的 市场需求是随机的且易受突发事件影响而发生较大变化。假设生产商不存在生产能力受限而 影响供货问题，在供应链契约有效期限内无突发事件冲击时其产品边际成本保持不变。相关 符号及说明如表 1 所示:

表 1、相关符号及说明

\begin{tabular}{|c|c|}
\hline 符号 & 符号说明 \\
\hline$Q$ & 供应链的订货量 \\
\hline$Q_{J}^{\circ}$ & 无应急情况时集中决策下的最优供应链订货量 \\
\hline$Q^{\circ}, Q_{M}^{\circ}, Q_{D}^{\circ}, Q_{R}^{\circ}$ & $\begin{array}{l}\text { 无应急情况时分散决策下的最优供应链订货 } \\
\text { 量、最优生产商订货量、最优分销商订货量及最 } \\
\text { 优零售商销售量 }\end{array}$ \\
\hline$Q_{B}^{\circ}$ & 突发事件发生时的供应链最优订货量 \\
\hline$x, f(x), F(x)$ & $\begin{array}{l}\text { 无应急情况时的市场随机需求量及其概率密 } \\
\text { 度函数和累积分布函数, } \mathrm{F}(\mathrm{x}) \text { 连续可导且严格递 } \\
\text { 增, } F(0)=0, \bar{F}(x)=1-F(x)\end{array}$ \\
\hline$y, g(y) 、 G(y)$ & $\begin{array}{l}\text { 突发事件冲击下的市场随机需求量及其概率 } \\
\text { 密度函数和累积分布函数, } \mathrm{G}(\mathrm{y}) \text { 连续可导且单调 } \\
\text { 递减, } G(0)=0, \bar{G}(y)=1-G(y)\end{array}$ \\
\hline$u$ & $\begin{array}{c}\text { 无应急情况时的市场产品随机需求均 } \\
\text { 值, } u=E(x)\end{array}$ \\
\hline$u G$ & $\begin{array}{c}\text { 突发事件发生时的市场产品随机需求均值, } \\
u G=E(y)\end{array}$ \\
\hline$c M, c D, c S$ & $\begin{array}{l}\text { 生产商、分销商及零售商的单位边际成 } \\
\text { 本, } c=c M+c D+c S\end{array}$ \\
\hline$\theta_{1}^{M}, \theta_{1}^{D}, \theta_{1}^{R}, \theta_{1}$ & $\begin{array}{l}\text { 当 } Q>Q^{\circ} \text { 时, 生产及销售扩大给生产商、 } \\
\text { 分销商、零售商及供应链整体带来的单位成本, } \\
\theta_{1}^{M} 、 \theta_{1}^{D} \text { 及 } \theta_{1}^{R} \text { 都大于零 }\end{array}$ \\
\hline$\theta_{2}^{M}, \theta_{2}^{D}, \theta_{2}^{R}, \theta_{2}$ & $\begin{array}{l}\text { 当 } Q<Q^{\circ} \text { 时, 生产及销售缩减给生产商、 } \\
\text { 分销商、零售商及供应链整体处理剩余产品带来 } \\
\text { 的单位成本, } \theta_{2}^{M} \text { 、 } \theta_{2}^{D} \text { 及 } \theta_{2}^{R} \text { 都大于零 }\end{array}$ \\
\hline$\omega M, \omega D$ & $\begin{array}{l}\text { 生产商及分销商制定的单位产品批发价格, } \\
\text { 满足 } \omega M>c M \text { 和 } \omega D>\omega M+c D\end{array}$ \\
\hline$\omega_{1}^{M}, \omega_{1}^{D}$ & $\begin{array}{l}\text { 无契约约束时分散式决策下生产商及分销商 } \\
\text { 制定的单位产品批发价格 }\end{array}$ \\
\hline$z M, z D 、 z S$ & $\begin{array}{l}\text { 生产商、分销商和零售商的单位缺货成 } \\
\text { 本, } z=z M+z D+z S\end{array}$ \\
\hline$v$ & 单位产品残值, 满足 $v<c M+c D+c S<p$ \\
\hline
\end{tabular}

\section{3.稳定市场状态下的三级供应链协调分析}

供应链协调管理的手段之一是通过各种单一或联合契约去约束相关利益方的行为，力图 将分散决策下的“双重边际效应”及“牛鞭效应”降到最低，在各相关利益方的利益不仅都不降 低甚至有所提高的条件下，实现供应链整体效率最优化，即实现 Pareto 最优。无突发事件干 
扰时，利用回购及收益共享的组合式柔性契约可以实现这个目标，三级供应链亦如此。

推论 1 、当市场需求处于稳定状态无契约约束时，供应链集中决策下的的最优订货量满 足: $F\left(Q_{J}^{\circ}\right)=\frac{p-c+z}{p-v+z}$

推论 2、当市场需求处于稳定状态无契约约束时，供应链分散决策下的的最优订货量满 足: $\mathrm{F}\left(Q^{\circ}\right)=\frac{p-c^{R}+z^{R}-\omega_{1}^{D}}{p-v+z^{R}}, Q^{\circ}<Q_{J}^{\circ}$ 。

推论 3、供应链系统各成员签订基于回购及收益共享的组合式柔性契约, 在契约参数满 足 $\omega D=p-c R+z R-\gamma(p-v+z R-p D)$ 及 $\omega M=\phi w D-c D+z D-\gamma(\phi p D+z D)$ 时, 分散决策下 的三级供应链可以实现协调。

由上述分析可知，在供应链系统分散决策下，通过变动 $\phi$, 可以调整零售商收益外的系 统整体收益在生产商和分销商之间的分配，该三级供应链在基于回购及收益共享契约的组合 式柔性契约约束下能够实现协调。

\section{4.突发事件冲击对三级供应链协调的影响}

假设生产商在销售季到来前，由于没有预估到影响产品销售的突发事件，仍然依据稳定 市场状态下预测的最优订购量安排生产。当实际进入销售季时市场的产品销售受到突发事件 冲击且冲击较大，造成分销商和零售商调整订货量、供应商被动调整生产计划以适应市场变 化，这样给供应链各成员都带来了额外成本，市场需求量的分布函数从 $\mathrm{F}(\mathrm{x})$ 变成 $\mathrm{G}(\mathrm{y})$ 。

供应链系统的期望利润函数 $(E \Pi(Q))$ 由于受突发事件变为:

$$
E \prod^{*}(Q)=p E_{G}(Q)+v E_{G}^{1}(Q)-c Q-z E_{G}^{2}(Q)-\left(\theta_{1}^{D}+\theta_{1}^{M}\right)\left(Q-Q_{B}^{\circ}\right)^{+}-\left(\theta_{2}^{R}+\theta_{2}^{D}+\theta_{2}^{M}\right)\left(Q_{B}^{\circ}-Q\right)^{+}
$$

根据 (3-1) 式, 突发事件发生时的供应链系统最优订货量 ( $\left.Q_{B}^{\circ}\right)$ 须满足: $Q_{B}^{\circ}=\arg ^{\max (Q>0)}\left(E \prod^{*}(Q)\right)$ 。

引理 1、如果突发事件给市场产品销售带来利好, 扩大了市场销售规模, 即 $\bar{G}(y) \geq \bar{F}(x)$, 则对于任意给定的 $Q \geq 0$, 都满足 $Q_{B}^{\circ} \geq Q^{\circ}$; 反之如突发事件给市场产品销售带来利空, 缩减 了市场销售规模，即 $\bar{G}(y) \leq \bar{F}(x) \mathrm{G}$ ，则对于任意给定的 $Q \geq 0$ ，都满足 $Q_{B}^{\circ} \leq Q^{\circ}$ 。

引理 2、产品的市场销售受突发事件影响而面临冲击时，供应链系统的订货策略需要调 整，这种情况下的最优供应链订购量须满足下式:

$$
Q_{B}^{\circ}=\left\{\begin{array}{c}
\bar{Q}, \bar{Q}>Q^{\circ} \\
Q^{\circ}, Q^{*} \leq Q^{\circ} \leq \bar{Q} \\
Q^{*}, Q^{*}<Q^{\circ}
\end{array}\right.
$$

上式中, $\bar{Q}$ 是突发事件冲击下, 市场产品销售扩大时满足 $E \Pi^{*}(Q)$ 一阶倒数为零时的最 优解 $\left(\bar{G}(Q)=\frac{c-v+\theta_{1}}{p-v+z}\right.$ 的解 $) ; Q^{*}$ 是市场产品销售缩减时满足 $E \Pi^{*}(Q)$ 一阶倒数为零时的最 优解 ( $\bar{G}(Q)=\frac{c-v-\theta_{2}}{p-v+z}$ 的解)。

由引理 1 及引理 2 可推导出以下定理:

定理 1、当突发事件对产品的市场销售产生冲击时，原来在无应急情况下制定的基于回 购及收益共享的组合式柔性契约无法保障三级供应链的协调。 
从上述三式可知，供应链各利益相关成员的期望利润函数不再是供应链系统整体期望利 润函数的线性函数, 该三级供应链在原来的联合契约约束下无法实现协调, $Q_{M}^{\circ} \neq Q_{D}^{\circ} \neq Q_{R}^{\circ} \neq Q_{B}^{\circ}$ 。

\section{5.突发事件干扰下供应链的协调分析}

前述分析表明，当突发事件对产品的市场需求产生冲击时，原来在稳定市场状态下制定 的组合式柔性契约无法保障三级供应链的协调。因此需要结合市场实际情况调整原有的组合 式柔性契约，使供应链系统重新达到协调。

柔性契约调整的出发点在于，根据既有的基于回购及收益共享的柔性联合契约特点，通 过调整契约参数来实现供应链系统的重新协调。

$$
\begin{gathered}
\text { 取 } \omega^{D}=p^{D}+v-c^{R}+\lambda^{3}\left(c-v+\frac{\lambda^{3} \theta_{1}\left(Q-Q^{\circ}\right)^{+}+\left(\lambda^{3} \theta_{2}-\theta_{2}^{R}\right)\left(Q^{\circ}-Q\right)^{+}}{\lambda^{3} Q}\right) \text { 及 } \\
\omega^{S}=\phi \omega^{M}-\phi p^{D}-c^{D}+\lambda^{2}\left(c-v+\frac{\left(\lambda^{2} \theta_{1}-\theta_{1}^{D}\right)\left(Q-Q^{\circ}\right)^{+}+\left(\lambda^{2} \theta_{2}-\theta_{2}^{D}\right)\left(Q^{\circ}-Q\right)^{+}}{\lambda^{2} Q}\right) \text { 。得出以下定理: }
\end{gathered}
$$

定理 2: 当突发事件对产品的市场需求产生冲击时，通过调整回购及收益共享契约参数 能使得三级供应链系统在新的组合式柔性契约约束下实现协调。

引入新组合式柔性契约后, 生产商、分销商及零售商的期望利润都为供应链整体利润的 线性函数, 满足 $Q_{M}^{\circ}=Q_{D}^{\circ}=Q_{R}^{\circ}=Q_{B}^{\circ}$, 突发事件干扰下不协调的供应链重新实现了协调。

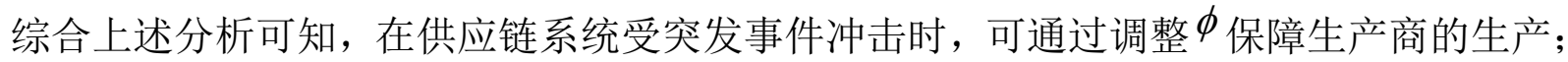
通过重新设定 $\omega M$ 及 $\omega D$, 在基于回购及收益共享契约的组合式柔性契约约束下实现供应链 系统的协调。

\section{6. 结论}

本文在一系列假设条件下，通过分析发现在市场受突发事件冲击较大时，会使得原有在 稳定市场状态下设置的组合式柔性契约无法保障供应链协调。这种情况出现的根本原因在 于随着突发事件的发生, 市场需求发生变化, 相应的市场随机需求函数也变化, 这些变化 影响了供应链契约环境, 如果相应的契约参数不变, 就会造成系统各成员从自身利益维护 出发进行 “逆向选择”，这样就会使得供应链不仅不再协调，甚至会导致其效率急剧下 降, 带来供应链中断风险。如果通过修改柔性契约的相关参数建立更富有弹性的供应链系 统, 重新对生产商、分销商和零售商之间的利润进行再分配, 实现一定程度上的风险共担 和利润共享，那么突发事件冲击下的供应链协调仍然在一定条件下可以实现。本文没有考 虑信息不对称及供应链成员风险偏好或厌恶的情况, 这些都是很有价值的下一步研究问题。

\section{References}

[1] Rameshwar Dubey, Angappa Gunasekaran, Stephen J. Childe, Thanos Papadopoulos, Samuel Fosso Wamba. World class sustainable supply chain management: critical review and further research directions[J]. The International Journal of Logistics Management . 2017 (2)

[2] Eva Niesten, Rodrigo Lozano. Making, buying and collaborating for more sustainable production and consumption[J] . Journal of Cleaner Production . 2015 